\title{
DNA's new colours
}

The current range of products for labelling DNA has many drawbacks: radioactive markers are a health hazard, and natural or chemical fluorescent proteins fade quickly, have a limited colour range and their overlapping absorption spectra make their combined use impractical. But Shuming Nie and colleagues, reporting in Nature Biotechnology, have solved the above problems by developing a sophisticated optical labelling system that relies on the unique properties of luminescent quantum dots (QDs) tiny light-emitting semiconductors.

QDs work like any other fluorophore they absorb light and re-emit it at a different wavelength. However, QDs are unique because the colour emitted by each one depends on its size, so QDs of any emission spectrum (that is, any colour) can be generated simply by varying the size of the QD particle. In addition, the QDs - brighter than conventional fluorophores are resistant to photobleaching, and QDs of different colours can be excited by the light of a single wavelength. Furthermore, unlike ordi-

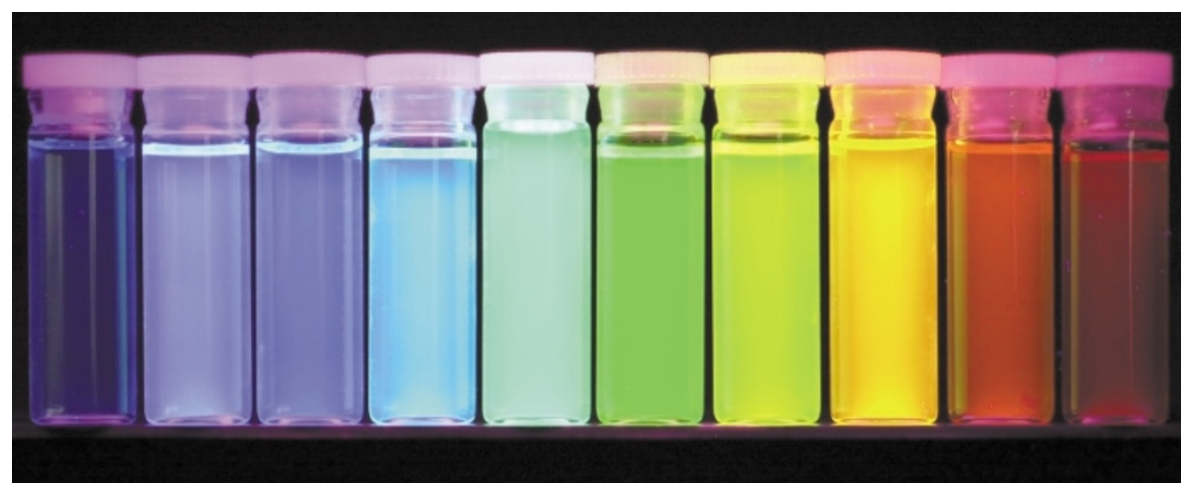

Image provided by Shuming Nie, Indiana University. @ (2001) Macmillan Magazines Ltd.

nary fluorophores, the emission spectra of individual QDs are very narrow, so QDs of different sizes can be used in combination by embedding them in polymer beads at precisely controlled ratios - effectively forming a bar-coded ID. As each colour can be manipulated to have different emission intensities, the number of different bar-coded beads that can be generated increases exponentially into the tens of thousands.

So what are the biological applications for these nifty new beads? Uniquely identifiable QD beads can be conjugated onto oligonucleotide probes or antibodies, opening new roads for medical diagnostics, drug screening and gene expression studies. DNA-chip experiments could be run more cheaply, effectively and quickly if the DNA probes were individually coded by beads that, as Nie and co-workers show, can be identified with an accuracy of 99.99\%. 'Rainbow' DNA chips might not be long in coming.

(2) References and links

Tanita Casci ORIGINAL RESEARCH PAPER Han, M. et al. Quantum-dottagged microbeads for multiplexed optical coding of biomolecules. Nature Biotechnol. 19, 631-635 (2001) FURTHER READING Rosenthal, S. J. Bar-coding molecules with fluorescent nanocrystals. Nature Biotechnol. 19, 621-622 (2001)

WEB SITE Shuming Nie's lab

\section{Nodal from top to bottom}
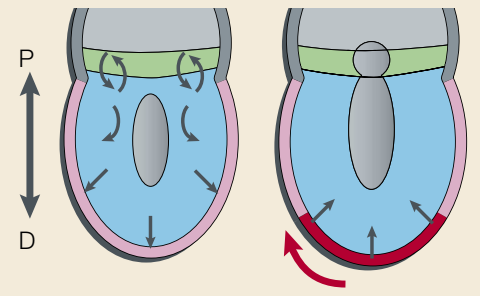

The anterior-posterior $(\mathrm{A} / \mathrm{P})$ axis is established very early in mouse embryogenesis, and a key molecule required for its formation, particularly for posterior cell fates, is Nodal - a member of the transforming growth factor- $\beta$ family of signalling molecules. New research shows that Nodal also establishes anterior cell fates through a distinct signalling mechanism. Before gastrulation, the mouse embryo exists as a radially symmetrical cup, comprising the epiblast (blue in the figure), which gives rise to the embryo itself, and two extra-embryonic tissues - the proximally located extra-embryonic ectoderm (ExE; green) and the visceral endoderm (pink).

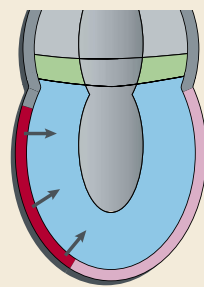

Coordinated cell movements convert proximal-distal $(\mathrm{P} / \mathrm{D})$ polarity into the $\mathrm{A} / \mathrm{P}$ axis. During this process, the visceral endoderm at the base of the cup rotates to one side to become the anterior visceral endoderm (AVE; red) and signals to the epiblast to repress posterior markers. In

particular, the AVE antagonizes the action of Nodal, which is required for the formation of posterior cell fates. Smad2 (Madh2) acts downstream of Nodal in the signal transduction pathway and is also required for $\mathrm{A} / \mathrm{P}$ axis formation, a result that was thought to reflect the action of Nodal and Smad2 in the same signalling pathway.

Brennan et al. have now discovered that although Nodal-or Smad2-null mutants both fail to form the $\mathrm{A} / \mathrm{P}$ axis, there are some important differences between them. The ExE is correctly patterned in Smad 2 mutants, but Nodal mutants rapidly lose expression of ExE markers. In addition, by studying various markers that are specific to the posterior of the embryo, they show that
Nodal mutants fail to express posterior markers, whereas Smad2 mutants express posterior markers throughout the epiblast. The authors explain this by showing that Nodal initially acts independently of Smad 2 in the epiblast to promote its own expression and that of posterior markers. By contrast, Smad2-dependent Nodal signalling from the epiblast to the visceral endoderm not only promotes Nodal expression but also is required for the formation of the AVE. Loss of Nodal therefore abolishes both the posterior and the anterior cell fates; loss of Smad2 abolishes only the anterior cell fate; and loss of either abolishes the $\mathrm{A} / \mathrm{P}$ axis.

Overall, these results highlight the importance of reciprocal interactions between extra-embryonic and embryonic tissues in patterning the early embryo. In particular, the paper suggests that Nodal acts in two different ways at the proximal and distal ends of the epiblast and is required, through independent mechanisms, for both anterior and posterior cell fates. But what initiates nodal signalling in the epiblast? The authors speculate that signals from the $\mathrm{ExE}$ are responsible - future work should tell. Mark Patterson

(2) References and links

ORIGINAL RESEARCH PAPER Brennan J. et al. Nodal signalling in the epiblast patterns the early mouse embryo. Nature 411, 965-969 (2001) 